through synaptic knobs on their surfaces and special spikes on their dendrites, whereby potential synaptic activity must be increased to an incredible extent. Much cerebral activity is concerned with the analysis of afferent stimuli in relation to previous experiences composed of memories and their emotional associations. This development of memory and the recog. nition of the familiar is believed to be effected through a process of neuronal facilitation with the ultimate result that what has been done once can more easily be done again. Intelligence presumably depends upon the capacity of the mind to associate together, rapidly and effectively, vast numbers of such memories, and this can only be if the brain is in a state of what amounts to almost ceaseless neuronic activity. Attention, memory, habit-formation, learning and intelligence all have their emotional aspects which are supremely powerful during early life, and it is the successful control, refinement and integration of these qualities that make possible the subsequent development of the balanced adult personality with perhaps unforseeable possibilities for the future. Considerations of speech and of such pathological states as epilepsy, pain and phantom limb sensations all yield additional information concerning the sensory-motor aspects of the brain-mind relationship.

This stimulating, provocative and at times speculative book is eminently readable and warmly to be recommended, especially for the wisdom of its concluding chapters.

Gilbert Hall

\section{YEARS OF LEARNING THEORY}

Theories of Learning

By Prof. Ernest R. Hilgard. Second edition. Pp. ix +563. (London: Methuen and Co., Ltd., 1959.) 45s. net.

THE first edition of this book appeared in 1948 .

In the intervening years, a considerable amount of experimental and theoretical effort has been devoted by American psychologists to the study of learning with, so it seems, no very radical advances being achieved. The work begins with E. L. Thorndike and proceeds by way of chapters on the major stimulus-response theorists, notably Skinner and Hull, and on the other group of theorists which the author designates the cognitive. The latter include those making use of gestalt conceptions with their implication of an emergence of qualitative differences in learning and problem-solving, as between lower and higher organisms, rather than a quantifiable dimension from simple to complex. These tend to infer more freely the existence of central brain (ideational) processes in accounting for observed behaviour modifications, whereas the former tend to explain in terms of peripheral intermediaries such behaviour as the goal-directed sequence of a rat running through a maze to a distant food-box.

The author makes clear that these two groups of theories each include a diversity of points of view around the central distinguishing characteristic of peripheral or central explanations. He points out that probably the major issue, which cuts across both groups, is that of the role of reinforcement and drive in learning. Some writers have supposed that, in the first instance, learning may occur simply through associative contiguity, the achieving of a reward or the avoiding of a punishment serving merely to facilitate the occurrence of the learned behaviour on subsequent occasions. Others have maintained that learning does not occur at all unless some kind of reinforcement is available to the organisms in the learning situation. The crux of the matter between these two points of view lies perhaps in the kind of definition given to the term 'reinforcement'. It need not consist merely in obtaining at primary goal, such as food or avoidance of electric shock, or secondary (acquired) goals such as counters or verbal praise or punishment. It may also derive from the reduction of tensions set going, for example, by the animal's propensity to explore its environment. The discussion of these problems, together with other current developments, including the role of mathematical models in the construction of learning theories, is contained in three new chapters which constitute an important addition to the first text.

Changes and additions to the historical core of the text are to be found in the chapter on Hull, where his later postulate system is assessed, in the discarding of the chapter on Wheeler's organismic psychology, and in the inclusion of a new chapter of 38 pages on Freud's psychodynamics. Wheeler's omission represents a loss of little significance, but it is not clear that Freud's irruption into a historical text dealing with theories derived almost entirely from experi. mental data constitutes a substantial gain. The author says: "If there is any teaching which has come from Freudian psychology it is that motives are organized in some sort of hierarchy within the individual, resulting in a value-system expressed in behaviour" (p. 348). But the key to this inclusion seems to be the awareness that motivation, whether in its conditioned or its instinctive form, is very inadequately dealt with by most learning theorists.

In considering the organisms from which learning data and theory are derived, it is common knowledge that the animal favoured by psychologists has been the rat. Index references confirm the rat's priority over all other laboratory animals in the ratio of about 2 to 1 . References to human learning make up a poor second to the rat. Allowing for the experimental conveniences of rats as learning-apparatuses, this still must constitute a severe restriction upon the general and comparative significance of the learning theories discussed. Indeed, it would seem justifiable to describe most stimulus-response theories as rat. derived, most cognitive theories as primate-oriented. This is a defect, not in the author's scope, but in the range of the work which he is very ably sampling. A vital corrective to this species-restriction is necessary, and much may be learned by learning theorists from the experimental-ethological studies of Thorpe, Tinbergen, and others.

We are left with the view that there may turn out to be several different types of learning which. at the present stage of theory construction and experimental evidence, cannot be resolved by single theory treatment, even of the promising mathematical model variety. But, although our reading of this book should lead to a critical dissatisfaction both with the theories and with the supporting evidence, the author has, in general, produced an invaluable historical survey and appreciation of current trends which merits the careful study of every student of behaviour. K. R. L. HALI 